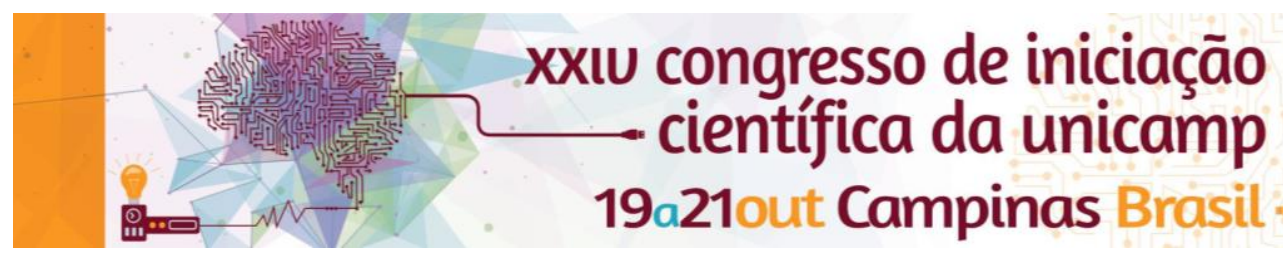

\title{
Tangible Interfaces for Programming Environments for Children
}

\author{
Maria Cecília Calani Baranauskas, Viviane Catini Nishiyama*.
}

\begin{abstract}
In the midst of the current technological developments, the concept of "Computational Thinking" (CT) has been discussed as a fundamental component for future generations. A ludic way to introduce CT is through new visual programming environments and platforms, and through the usage - yet to be explored - of tangible interfaces for the access of such environments. This research project aims: to analyze the role of these systems and their interfaces (focusing on the programs Kodu and Scratch) in the educational context; to develop supportive materials, for teachers and students, to help on the usage of the environments; and to conduct an experiment, involving all systems, with children attending a public school of Campinas, comparing its results to what was predicted according to the literature.
\end{abstract}

\section{Key words: \\ Computational Thinking, tangible interfaces, visual programming environments for children.}

\section{Introduction}

As a pioneer in the concept of CT, Wing describes it as a way of "solving problems, designing systems, and understanding human behavior, by drawing on the concepts fundamental to computer science" [1]. The introduction of this kind of logic in the first years of study has many benefits, and can be accomplished by teaching programming concepts with the support of graphic and tangible platforms developed specially for children.

Under these circumstances, this research project aimed to analyze the role of these systems and their interfaces (focusing on the programs Kodu and Scratch) in the educational context, and to compare them with each other. Moreover, it was also an aim to create supportive materials, for teachers and students, and to develop demo applications based on the environments and tangible interfaces. Subsequently, involving all systems, an experimentation was conducted with children attending a public school of Campinas, and its results were compared with what was predicted according to the literature and what was previously expected.

\section{Results and Discussion}

After searching the literature and analysing its results, two manuals were developed, explaining how to access and how to interact with each of the graphic platforms - Kodu and Scratch. Using a lot of images and a simplified language, these materials were made to be accessible to teachers and students without previous knowledge of the platforms.

Regarding the tangible interfaces, this research was based on two interfaces developed by graduate students of UNICAMP. The first is a platform for storytelling named Computation Programmable Environment for Storytelling (CPES); while the second, Tangible Programmable Environment for Children (TaPrEC), comprises wooden pieces that can compose lines of programming code when put together. For both environments, manuals and application demos were created.

A research group performed CPES's experimentation in a set of educational units of UNICAMP named DediC "Divisão de Educação Infantil e Complementar". While another group performed TaPrEC's experimentation in a supplementary educational program named PRODECAD - "Programa de Integração e Desenvolvimento da Criança e do Adolescente". In both moments, the groups conducted weekly workshops for teachers and children.

Generally, it was noticed that tangible environments and their technologies aroused curiosity and excitement among children. Some commands and actions were easily comprehended, whereas others showed a level of difficulty. The presence of a guide to explain about the exercises appeared to be essential, particularly for those tasks that involved logic challenges or abstract programming skills. A few teachers had difficulty with the technology being used.

\section{Conclusions}

In agreement with the literature, the tangible tools aroused a great interest in the users, while teaching concepts related to Computational Thinking. Both of the mentioned tangible environments have a relatively low cost, making them affordable, and can be used as a great tool to create multiple exercises and activities. Considering all of these positive characteristics, this kind of entertaining and ludic tool has the potential to enrich the education of children and to better prepare them in this era of computational development.

\section{Acknowledgement}

I would like to sincerely thank my supervising professor, Cecília Baranauskas, and the graduate students, Julian Posada e Marleny Carbajal, for all the guidance and support before and during my undergraduate research.

This research was financially supported by CNPq.

1 Wing, J.M. Computational thinking, Communications of the ACM, 2006, v. 49 , n. 3 